\title{
Visiones del Diseño I: El Diseñador como agente de cambio Proyecto de Investigación \\ $\mathrm{N}^{\circ} 4.2$ \\ Equipo de Investigación ${ }^{(1)}$ \\ Daniela V. Di Bella \\ Terry Irwin \\ por School of Design at Carnegie Mellon (EEUU)
}

por Facultad de Diseño y Comunicación, Universidad de Palermo (ARG)

\begin{abstract}
Resumen: El Proyecto de Investigación 4.2 sostiene que el diseño en la actualidad se encuentra inserto dentro de múltiples escenarios de complejidad que requieren de profesionales de fuerte conocimiento de las implicancias de su disciplina y de una amplia capacidad reflexiva. En este contexto investiga acerca del estado actual del Diseño siguiendo cuatro categorías temáticas que cubren algunos de los escenarios contemporáneos de la disciplina en la región, relacionados con (a) reflexiones sobre diseño, (b) diseño sustentable, (c) transición hacia la sostenibilidad y (d) Diseño para la transición. El apartado (d) Diseño para la Transición, analiza un informe de impacto retrospectivo y continuo, de la implementación del Primer Proyecto de la Línea de Investigación No4 denominado Perspectivas del Diseño, que analiza el período 2014_2 al 2016_2 inclusive, correspondiente con las comisiones de la asignatura Diseño IV de la Maestría en Gestión del Diseño, en la que se viene desarrollando el proyecto.
\end{abstract}

Palabras clave: Diseño en perspectiva - Escenarios del Diseño - Responsabilidad del Diseño - Futuro del Diseño - Diseño emergente - Diseño para la Transición - Diseño sostenible - Transiciones socio-técnicas - Investigación en diseño - Teoría del diseño.

[Resúmenes en inglés y portugués en la página 138]

${ }^{(1)}$ Los CVs del Equipo de Investigación pueden consultarse en el Capítulo Directores de Líneas y Coordinadores de Proyectos de esta misma Edición. 


\section{Acerca del Proyecto 4.2}

\section{Visiones del Diseño I \\ El Diseñador como agente de cambio}

El Proyecto 4.2 Visiones del Diseño I: El Diseñador como agente de cambio, aborda una mirada integral del diseño considerándolo desde una perspectiva sistémica que revisa los escenarios contextuales en todas sus dimensiones y capas de incidencia haciendo foco sobre la relación Sociedad-Economía-Ambiente del hacer proyectual del Diseño y de la Arquitectura. Propone una exploración y reflexión que pretende enriquecer el campo teórico del diseño y sus espacios de conceptualización disciplinar, a partir de la noción de un diseño que se gestiona y desarrolla desde las fronteras de su relación con otras disciplinas en un vínculo inter/transdisciplinar que amplifica y expande los marcos tradicionales de su ejercicio y aplicación a la realidad local y de la región.

Manifiesta la necesidad de anticiparse y planificar escenarios a largo plazo que promuevan transiciones sociales hacia futuros sostenibles, donde el diseño y los diseñadores puedan constituirse en agentes de cambio. Relaciona al Diseño con el concepto de transición, y de las sociedades en transición, asunto que representa un marco pluri-ideológico que instala un espacio del debate y cuestionamiento vinculado con la gestación de una transformación cultural relativa a la cosmogonía global que rige el ordenamiento y estructuración política, social y económica de los últimos 200 años.

A partir del Acuerdo de Cooperación Académica celebrado en 2014, entre la School of Design at Carnegie Mellon University y la Universidad de Palermo, la Facultad de Diseño y Comunicación incorpora una nueva línea de exploración, reflexión e investigación denominada Diseño en Perspectiva vinculada al Programa Diseño para la Transición (Transition Design) que esta prestigiosa Universidad desarrolla a nivel de Doctorado y Maestría en Estados Unidos, para avanzar en una investigación en la que participan académicos de ambas Instituciones, con la coordinación compartida de Daniela V. Di Bella (UP) y Terry Irwin (CMU), con el fin de Impactar sobre la configuración del diseño y la arquitectura, hacia la orientación del ejercicio profesional basado en buenas prácticas relacionadas con la responsabilidad social y ecológica del diseño en los planos regional y global. Las reflexiones y los resultados obtenidos en el Proyecto 4.2 Visiones del Diseño I. El Diseñador como agente de cambio, son continuación del Proyecto 4.1 Perspectivas del Diseño, El diseño en nuevos escenarios disciplinares, y se continúan desarrollando en los Proyectos 4.3 Visiones del Diseño II: Diseñadores Eco-Sociales, y 4.4.Visiones del Diseño III: Problematizar el Diseño para comprender su complejidad, en el marco de la misma Línea de Investigación y bajo la misma Directora Daniela V. Di Bella.

Sus principales objetivos son:

- Reflexionar y orientar a los profesionales del ámbito de los campos proyectuales, hacia el ejercicio de buenas prácticas profesionales, relacionadas con la responsabilidad social y ecológica del diseño en los planos regional y global. 
- Amplificar los ámbitos de análisis del diseño, a través de una comprensión holística que lo considera desde una perspectiva sistémica que revisa sus escenarios contextuales en todas sus dimensiones y capas de incidencia.

- Instalar la práctica reflexiva, exploratoria y analítica que conjuga Diseño con los estudios para la Transición, con el fin de generar visiones de diseño anticipatorias sostenibles y de largo alcance para la solución de problemas de Diseño y gestión.

- Fomentar la inter/transdisciplina en el ejercicio académico y profesional, a partir de la noción de un diseño que se gestiona y desarrolla desde las fronteras de su relación con otras disciplinas, situación que expande los marcos tradicionales de su ejercicio y aplicación.

- Impactar sobre la graduación de generaciones de diseñadores para que sean capaces de trabajar eficazmente en la solución de problemas complejos, insertos en equipos transdisciplinarios y transversales cuyas propuestas contribuyan con gestar transiciones sociales positivas y sostenibles.

\section{Acerca de la Línea 4}

Diseño en Perspectiva: Escenarios del Diseño dirigida por Daniela V. Di Bella se desarrolla de manera ininterrumpida desde 2014 en la Facultad de Diseño y Comunicación (UP, Argentina), e incluye hasta el momento cuatro proyectos finalizados el 4.1 Perspectivas del Diseño, 4.2 Visiones del Diseño I: El Diseñador como agente de cambio, 4.3 Visiones del Diseño II: Diseñadores Eco-Sociales, 4.4.Visiones del Diseño III: Problematizar el Diseño para comprender su complejidad, coordinados por Daniela V. Di Bella (UP) y Terry Irwin (CMU).

\section{Mapa de Áreas y Proyectos}

El Proyecto 4.2 está vinculado a la asignatura Diseño IV que dicta Daniela V. Di Bella de la Maestría en Gestión del Diseño (UP), guarda relación con todas las carreras del Diseño y la Arquitectura en grado, posgrado y sus áreas de influencia; con el Programa Transition Design de la School of Design at Carnegie Mellon (USA) que se desarrolla a nivel de Doctorado en esa institución, y con el Transition Design Institute de la misma Institución académica dirigido por Terry Irwin. Por su actualidad e importancia impacta no solo sobre el posgrado al que se vincula, sino sobre todas las carreras de grado y posgrado en diseño y arquitectura que analicen y reflexionen sobre los escenarios presentes y futuros de la disciplina en los planos regionales y globales. 


\section{Productos y Resultados}

\section{a)- Publicaciones}

Cuaderno del Centro de Estudios de Diseño y Comunicación No80. (2020) Diseño en Perspectiva - Diseño para la transición. Segunda Sección. Coordinación Daniela V. Di Bella (UP) y Terry Irwin (CMU). Facultad de Diseño y Comunicación Año XXI, Buenos Aires, Argentina. ISSN: 1668-0227. Esta publicación documenta y comunica los resultados alcanzados en el proyecto de investigación 4.1 Perspectivas del Diseño y da inicio al proyecto 4.2 Visiones del Diseño I, y a continuación se detallan los autores y artículos contenidos en ella:

Barón Gabriela N. (2020) La transición urbana y social hacia un paradigma de movilidad sostenible (Pp. 153-172)

Di Bella, Daniela V. (2020) Prólogo de la Segunda Sección. Diseño en Perspectiva - Diseño para la Transición. (Pp. 11-16)

Di Bella, Daniela V. (2020) Prefacio Diseño en Perspectiva (Pp. 17-22)

Di Bella, Daniela V. (2020) Impacto de la Experiencia Diseño en Perspectiva (Pp. 173-239)

González Insúa, Mariana. (2020) Más allá del producto: un abordaje local sobre el diseño de productos sistemas-servicios para la sustentabilidad y tecnologías de inclusión social (Pp. 91-109)

Marchisio, Mariela (2020) El fin de las escuelas de diseño (Pp. 51-63)

Moroni I, de Arruda Amilton J V (2020) Comprender cómo los procesos de diseño pueden contribuir a la mejora de la capacidad innovadora en el universo de las startup companies (Pp. 65-75)

Mouchrek N, Krucken L. (2020) Diseño como agente de cambio: iniciativas orientadas a la práctica en la enseñanza del diseño (Pp. 123-138)

Mouchrek N. (2020) Diseño para el desarrollo de la juventud y su participación en la sostenibilidad (Pp. 139-151)

Portugal do Nascimento, Luís C. (2020) Diseño en medio de feudos y campos: la oportunidad de la "rectificación de nombres" propuesta por Confucio en la Babel contemporánea de conceptos, términos y expresiones pegadizas recientemente forjados en el campo del diseño (Pp. 23-36)

Soto, Carlos. (2020) Esto No es Diseño (Pp. 37-50)

Stivale, Silvia. (2020) Los caminos del diseño sustentable y sus vinculaciones con la investigación en Diseño (Pp. 77-90)

Soares T, de Arruda Amilton J V. 2020) Domos geodésicos como modelo de negocio en la gestión hotelera para el desarrollo de las economías locales (Pp. 111-122)

Actas de Diseño No27. (2019) XIII Encuentro Latinoamericano de Diseño "Diseño en Palermo" IX Congreso Latinoamericano de Enseñanza del Diseño Comunicaciones Académicas. Facultad de Diseño y Comunicación, Universidad de Palermo. Buenos Aires, Argentina. En esta publicación se documentan se documentan las ponencias de la Comisión Diseño en Perspectiva correspondientes a la presentación de los Cuadernos 73 y 80 durante el III Coloquio de Investigación y Desarrollo en Diseño (Ponencias en Pp. 42-43 y 70). 
Actas de Diseño No24. (2018) XII Encuentro Latinoamericano de Diseño "Diseño en Palermo". Foro de Escuelas de Diseño - XI Plenario 2017, VIII Congreso Latinoamericano de Enseñanza del Diseño. II Coloquio de Investigación y Desarrollo en Diseño Latino. Coloquio de Investigación. Julio 2017, Buenos Aires, Argentina. En esta publicación se documentan las ponencias de la Comisión Diseño en Perspectiva correspondientes al período (2016_2/2017_1) presentadas en el II Coloquio de Investigación y Desarrollo en Diseño (Pp.34-36).

Actas de Diseño No22. (2017) XI Encuentro Latinoamericano de Diseño "Diseño en Palermo". Foro de Escuelas de Diseño - XI Plenario 2016, VII Congreso Latinoamericano de Enseñanza del Diseño. Julio 2016, Buenos Aires, Argentina. En esta publicación se documentan las ponencias de la Comisión Diseño en Perspectiva correspondientes al período (2015_2/2016_1) presentadas en el II Coloquio de Investigación y Desarrollo en Diseño. Ponencias (Pp. 69-71) y Conclusiones (Pp.100).

Actas de Diseño No20. (2016) X Encuentro Latinoamericano de Diseño "Diseño en Palermo". Foro de Escuelas de Diseño - X Plenario 2015, VI Congreso Latinoamericano de Enseñanza del Diseño. Julio 2015, Buenos Aires, Argentina. En esta publicación se documentan las ponencias de la Comisión Diseño en Perspectiva correspondientes al período (2014_2/2015_1) presentadas en el II Coloquio de Investigación y Desarrollo en Diseño. Ponencias (Pp. 70-71) y Conclusiones (Pp.100).

Escritos en la Facultad No122. (2016) Articulación entre Investigación y Posgrados, Edición 1. Doctorado en Diseño. Maestría en Gestión del Diseño. Programa de Investigación y Desarrollo en Diseño Latino. Línea de Investigación No4: Diseño en perspectiva. El diseño en contextos de transición (Transition Design). En convenio con School of Design at Carnegie Mellon University, EEUU. ISSN: 1669-2306 ISSN (En línea): 2591-3743. En esta publicación la Directora Daniela V. Di Bella presenta la línea de investigación No4 Diseño en Perspectiva: escenarios del diseño y anuncia el comienzo del Proyecto 4.1 Perspectivas del Diseño (Pp. 10 y 21-22).

\section{b)- Congresos / Coloquios / Plenarios}

$2^{\circ}$ Plenario de Directores de Investigación DC, 27 y 4 de septiembre - 2018. Es este segundo plenario los Directores de las Líneas y Proyectos de Investigación presentaron a sus pares y al conjunto del Programa de Investigación de la Facultad de Diseño y Comunicación, los resultados obtenidos y/o en proceso (publicaciones y acuerdos), junto con los avances de Proyectos. Es esta ocasión la Directora de la Línea presentó los resultados obtenidos en el Primer proyecto de Investigación (4.1) Perspectivas del Diseño y anunció el comienzo del segundo proyecto 4.2 Visiones del Diseño I junto con sus objetivos 20192020 y sus acciones en desarrollo. 
III Coloquio de Investigación y Desarrollo en Diseño Latino. Universidad de Palermo, 30 de julio de 2018. IX Congreso Latinoamericano de Enseñanza del Diseño. Semana Internacional del Diseño en Palermo.

En la comisión Diseño en perspectiva se presentaron las conclusiones del proyecto 4.1 Perspectivas del Diseño, y se dio inicio al segundo proyecto de la línea 4.2 Visiones del Diseño I. Expusieron: Daniela V. Di Bella, Mariela Marchisio, Mariana González Insúa, Carlos Soto, Silvia Stivale, Theska Soares, Amilton Arruda, e Isabel Moroni.

A continuación se detallan las ponencias presentadas en esta Comisión coordinada por la Directora del proyecto Daniela V. Di Bella:

Di Bella, Daniela V. (Argentina) - Coordinadora de la Comisión

Visiones del Diseño: Presentación de los Cuadernos 73 y 80, y $2^{\circ}$ Proyecto de la Línea CMU-UP

Marchisio, Mariela (Argentina)

Los nuevos desafíos de la educación en Diseño

Gonzalez Insúa, Mariana (Argentina)

Producto Sistema Servicio para la Sustentabilidad y Tecnologías de Inclusión Social. Una aproximación interdisciplinar a la sustentabilidad urbana.

Soto, Carlos (Colombia)

Definiendo el diseño

Stivale, Silvia (Argentina)

Diseño con intención hacia conductas sustentables

Soares Theska, Arruda Amilton (Brasil)

Design Estratégico: O estudo de casos relacionados ao uso da metodologia do Biomimicry Thinking

Moroni, Isabela, Arruda Amilton (Brasil)

Processos de design: uma abordagem teórica para inovação em empresas startups

$1^{\circ}$ Plenario de Directores de Investigación DC, 13 de octubre - 2017. Es este primer plenario los Directores de Líneas y Proyectos de Investigación presentaron a sus pares y al conjunto del Programa de Investigación de la Facultad de Diseño y Comunicación, los resultados obtenidos y/o en proceso (publicaciones y acuerdos), junto con los Proyectos.

La Directora de la Línea de Investigación No4 presentó los principales avances del Proyecto 4.1 Perspectivas del Diseño, entre ellos:

- las gestiones por las publicaciones en proceso editorial (Cuadernos 73 y 80 ) y los profesionales invitados por ambas instituciones (CMU y UP);

- los detalles de la adaptación curricular y tratamiento temático en el aula de Diseño IV de Maestría en Gestión del Diseño y sus principales repercusiones;

- el resumen conceptual de las ponencias de estudiantes de Maestría presentadas en las comisiones Diseño en Perspectiva 2015, 2016 y 2017 durante los Encuentros, Congresos y Coloquios respectivos;

- la proyección de la línea hacia un segundo proyecto 4.2 Visiones del Diseño I tendiente a consolidar las ideas en estudio. Esta exposición deriva luego en el informe de impacto contenido en el Cuaderno 80. 
Di Bella, Daniela V. (2018) Impacto de la Experiencia Diseño en Perspectiva.

II Coloquio de Investigación y Desarrollo en Diseño Latino. Universidad de Palermo, 31 de julio de 2017. VIII Congreso Latinoamericano de Enseñanza del Diseño. Semana Internacional del Diseño en Palermo.

En la comisión Diseño en perspectiva se presentaron las reflexiones y avances del proyecto en desarrollo 4.1 Perspectivas del Diseño, expusieron: Daniela V. Di Bella, José María Mazzei, Paola M. Trocha Sánchez, Marina Cordova, Daniel Garrido, Cindy Mera, Maria Amor Buiras y los ponentes invitados Gabriela Nuri Baron, Silvia Stivale, Theska Laila y Amilton Arruda.

A continuación se detallan las ponencias presentadas en esta Comisión coordinada por la Directora del proyecto Daniela V. Di Bella:

Ponencias de la Comisión 4, Diseño en Perspectiva* correspondientes al período (2016_2/2017_1).

Di Bella, Daniela V. (Argentina) - Coordinadora de la Comisión

Impacto de la Experiencia Diseño en Perspectiva

Mazzei, José Maria (Argentina)*

Tendencia ecologista y Diseño en Argentina

Trocha Sánchez, Paola Marcela (Colombia)*

Sombrero Vueltiao: transiciones de un objeto artesanal

Cordova, Marina (Bolivia)*

Las campañas de comunicación visual como agentes de cambio social-ambiental

Garrido, Daniel (Ecuador)*

Demandas contemporáneas del Diseño de Interiores

Mera, Cindy (Ecuador)*

Diseño universal y accesibilidad web

Buiras, María Amor (Argentina)*

El branding y la representación social del cuerpo en la posmodernidad

*Ponencias de los trabajos reflexivos desarrollados para la presente Línea de Investigación en la asignatura Diseño IV de la Maestría en Gestión en Diseño

Ponentes invitados de otras Universidades

Nuri Baron, Gabriela (Argentina)

La transición urbana y social hacia un paradigma de movilidad sostenible

Stivale, Silvia (Argentina)

Los caminos del Diseño Sustentable y sus vinculaciones con la investigación en diseño Laila Theska, Arruda Amilton (Brasil)

Geodesic Domes as Business Model in Hotel Management for Local Economies Development 
I Coloquio de Investigación y Desarrollo en Diseño Latino. Universidad de Palermo, 26 de Octubre de 2016.

Se presentó la Línea de Investigación №4: Diseño en Perspectiva, Escenarios del Diseño, como una de las líneas que integran el Programa de Investigación en Diseño de la Facultad de Diseño y Comunicación.

Di Bella, Daniela V. (Argentina) Presentación de la Línea de Investigación Nº4: Diseño en Perspectiva, Escenarios del Diseño. Bajo el convenio de cooperación académica entre Facultad de Diseño y Comunicación, Universidad de Palermo y School of Design at Carnegie Mellon University.

VII Congreso Latinoamericano de Enseñanza del Diseño (Julio 2016): Presentación de las Ponencias de la Comisión Diseño en Perspectiva* correspondientes al período (2015_2/2016_1) (Maestría y Licenciatura en Diseño).

Presentaron sus avances Daniela V. Di Bella, Andrea Pontoriero, Naylé Lombide, Sofía Zurita, David Ortega, Sasha Santa María Salas, Melanie Etse, Kelly David Lopez, Carolina Gutierrez Ferreira y Sandra Beltrán.

A continuación se detallan las ponencias presentadas en esta Comisión coordinada por la Directora del proyecto Daniela V. Di Bella:

Di Bella, Daniela V. (Argentina)

Experiencia Diseño en Perspectiva

Pontoriero, Andrea (Argentina)*

Arte $y$ diseño: simulacro, perfomance y sociedad

Lombide, Naylé (Cuba)*

Cultura de los objetos en Cuba

Zurita, Sofía (Ecuador)*

Diseño inclusivo en Ecuador

Ortega, David (Colombia)*

Drap-Art, Cuando la consigna no es vender

Santa María Salas, Sasha (Ecuador)*

El discurso creativo del movimiento maker: artesanía y digitalidad

Etse, Melanie (Argentina)*

Gestión de diseño en campañas antitabaco en Buenos Aires

David López, Kelly (Colombia)*

Hacia una marca ciudad: Caso Centro histórico de Pasto Colombia

Gutierrez Ferreira, Carolina (Colombia)*

Mobiliario objeto de consumo: Caso Ikea

Beltrán, Sandra (Ecuador)*

Modelos de gestión para el diseño de autor en Quito, Ecuador

*Ponencias de los trabajos reflexivos desarrollados para la presente Línea de Investigación en la asignatura Diseño IV de la Maestría en Gestión en Diseño 
VI Congreso Latinoamericano de Enseñanza del Diseño (Julio 2015): Creación de la Comisión Diseño en Perspectiva y Presentación de Ponencias* correspondientes al período (2014_2/2015_1).

Presentaron sus avances Daniela V. Di Bella, Ismael Bonoff, Washington da Silva Neves, Luciana Finkielsztoyn, José Luis Oviedo, Paulina Godoy y Macarena Faust.

A continuación se detallan las ponencias presentadas en esta Comisión coordinada por la Directora del proyecto Daniela V. Di Bella:

Di Bella, Daniela (Argentina)

Presentación del Proyecto Diseño en Perspectiva

Bonoff, Ismael (Argentina)*

Desafíos del Diseñador Industrial en la Era del Consumo

da Silva Neves, Washington (Brasil)*

Diseño Kitsch y compromiso social del Diseñador

Finkielsztoyn, Luciana (Argentina) ${ }^{*}$

Emoción versus serialidad. Diseño de autor y mass fashion

Oviedo, José Luis (Perú)*

Geometría cultural: víctimas de una mentalidad mediatizada

Godoy, Paulina (Chile)*

Identidad Chilena y Joyería de Autor

Faust, Macarena (Argentina)*

La Indumentaria que habla

* Ponencias de los trabajos reflexivos desarrollados para la presente Línea de Investigación en la asignatura Diseño IV de la Maestría en Gestión en Diseño

\section{c)- Formación de Posgrado e Impacto curricular}

La Directora Daniela V. Di Bella es Docente de Posgrado en Diseño de la Facultad de Diseño y Comunicación en Maestría en Gestión del Diseño y Doctorado en Diseño y al igual que Terry Irwin en su institución (CMU), ambas incorporan los contenidos de su investigación a sus asignaturas en posgrado.

La incorporación de la temática Diseño para la Transición en la asignatura Diseño IV de la Maestría en Gestión en Diseño, y las acciones desarrolladas y proyectadas en los períodos (2014_2-2017_1 y 2017_2/2019), proporcionan datos de influencia sobre la aplicación de producción teórica y reflexiones en trabajos prácticos y campo profesional de los estudiantes de posgrado (Maestría y Doctorado) y grado (Proyectos de graduación) verificables, entre otras, en el tratamiento curricular de la asignatura Diseño IV implementado desde $2014 \_2$ a la fecha; y en las ponencias presentadas en las Comisiones Diseño en Perspectiva $2015,2016,2017,2018,2019$ y 2020 en el $6^{\circ}, 7^{\circ}, 8^{\circ}, 9^{\circ}, 10^{\circ}$ y $11^{\circ}$ Congreso Latinoamericano de Enseñanza del Diseño respectivamente juntamente con el I, II, III, IV y V Coloquio de Investigación y Desarrollo en Diseño Latino. (Ver informes descriptivos que se citan a continuación) 
Di Bella, Daniela V. (2018) Impacto de la Experiencia Diseño en Perspectiva. En Cuaderno del Centro de Estudios de Diseño y Comunicación No80. (Impreso 2020) Diseño en Perspectiva - Diseño para la transición. Segunda Sección. Coordinación Daniela V. Di Bella (UP) y Terry Irwin (CMU). Facultad de Diseño y Comunicación Año XXI, Buenos Aires, Argentina. ISSN: 1668-0227. (Pp. 173-239)

Di Bella, Daniela V. (2017) Diseño en Perspectiva-Diseño para la Transición - Prólogo. En Cuaderno del Centro de Estudios de Diseño y Comunicación No73. (Impreso 2019) Diseño en Perspectiva - Diseño para la transición. Primera Sección. Coordinación Daniela V. Di Bella (UP) y Terry Irwin (CMU). Facultad de Diseño y Comunicación Año XIX, Buenos Aires, Argentina. ISSN: 1668-0227. (Pp. 13-18)

\title{
Evaluación Externa
}

El Proyecto 4.2 Visiones del Diseño I: El Diseñador como agente de cambio, con la School of Design at Carnegie Mellon (EEUU), cuyos resultados fueron publicados en el Cuaderno No80, fue evaluado exitosamente por la Evaluadora Jimena Ascolani en 2019.

\begin{abstract}
Research Project 4.2 maintains that design is currently inserted within multiple complexity scenarios that require professionals with a strong knowledge of the implications of their discipline and a broad reflective capacity. In this context, he investigates about the current state of Design following four thematic categories that cover some of the contemporary scenarios of the discipline in the region, related to (a) reflections on design, (b) sustainable design, (c) transition towards sustainability and (d) Design for the transition. Section (d) Design for Transition, analyzes a retrospective and continuous impact report of the implementation of the First Project of Research Line No. 4 called Design Perspectives, which analyzes the period 2014_2 to 2016_2 inclusive, corresponding to the commissions of the Design IV subject of the Master in Design Management, in which the project is being developed.
\end{abstract}

Keywords: Perspective Design - Design Scenarios - Design Responsibility - Future of Design - Emerging Design - Design for Transition - Sustainable Design - Socio-technical Transitions - Design Research - Design Theory.

Resumo: O Projeto de Pesquisa 4.2 sustenta que o design está atualmente inserido em cenários de múltiplas complexidades que requerem profissionais com forte conhecimento das implicações de sua disciplina e ampla capacidade reflexiva. Neste contexto, investiga o estado atual do Design a partir de quatro categorias temáticas que abrangem alguns dos cenários contemporâneos da disciplina na região, relacionadas com (a) reflexões sobre design, (b) design sustentável, (c) transição para a sustentabilidade e (d) Projeto 
para a transição. A seção (d) Desenho para Transição, analisa um relatório de impacto retrospectivo e contínuo da implementação do Primeiro Projeto de Linha de Pesquisa n 4 denominado Perspectivas de Desenho, que analisa o período de 2014_2 a 2016_2 inclusive, correspondendo às comissões de a disciplina de Design IV do Mestrado em Gestão de Design, em que o projeto está a ser desenvolvido.

Palavras chave: Design de perspectiva - Cenários de design - Responsabilidade do design - Futuro do design - Design emergente - Design para a transição - Design sustentável Transições sociotécnicas - Pesquisa em design - Teoria do design.

[Las traducciones de los resúmenes fueron realizadas a través de traductor automático] 